\section{Topographical layer imaging as a tool to track neurodegenerative disease spread in M 1}

\section{Stefanie Schreiber $(\mathbb{D})$, Alicia Northall $(\mathbb{D}$, Miriam Weber, Stefan Vielhaber and Esther Kuehn}

In their recent Review (McColgan, P., Joubert, J., Tabrizi, S. J. \& Rees, G. The human motor cortex microcircuit: insights for neurodegenerative disease. Nat. Rev. Neurosci. 21, 401-415 $(2020))^{1}$, McColgan et al. provide a comprehensive overview of the in vivo microcircuits of the human primary motor cortex (M1) and develop hypothetical models of M1 cell loss and layer-specific dysfunction in neurodegenerative disorders. Ultra-high-field (UHF) MRI is promoted in the article as a methodology to uncover the neuronal mechanisms of disease pathophysiology and disease spread in the living individual. This is particularly important given existing ex vivo methods can investigate neurodegeneration only at (usually very) advanced stages and after massive cell loss. Building on this, we here add to this argument that in vivo models on microstructural M1 degeneration can only be successfully developed if local inhomogeneities within $\mathrm{M} 1$ are taken into consideration.

The microarchitecture in M1 exhibits a surprisingly high heterogeneity even in healthy individuals and warrants a precise investigation. The M1 myeloarchitecture varies according to cortical depth (with deep layers being most densely myelinated) and along the cortical surface (for example, the area representing the hand is more myelinated than the area representing the face) $)^{2}$ (FIG. 1 a). In addition, there are boundaries of low myelin that separate major body part representations in M1, such as between the hand area and the face area, and that divide M1 into different cortical fields ${ }^{2,3}$. These heterogeneities may explain some of the variance in how neurodegenerative disorders spread through M1 and how neurodegenerative changes are visible in in vivo MRI. Three-dimensional models of cortical structure and cortical function (one dimension in cortical depth and two dimensions along the cortical surface ${ }^{4}$ ) could elucidate layer-wise, body-part-wise and hemisphere-wise disease spread and its accordance with motor phenotype and symptom progression (FIG. 1 b).

The authors discuss the possibility to use in vivo UHF MRI to characterize amyotrophic lateral sclerosis (ALS), a disorder that rapidly spreads through M1. However, the authors do not mention that symptom onset may be bulbar or lateralized according to the upper or lower limbs and therefore differs with respect to the topographical location in M1. The b Three-dimensional model of disease spread in M1 spread of motor symptoms commonly mirrors body-part-wise disease spread first across the ipsilateral and afterwards across the contralateral hemisphere ${ }^{5}$. With an assumed disease onset in cortical layer 5 (L5), as McColgan et al. suggest, ALS demands an investigation across three rather than two dimensions, to take into account inherent variations in the M1 microstructure. McColgan et al. suggest that, in ALS, myelin and cell loss spreads from L5 to L2/3 before affecting other cortical areas ${ }^{1}$ (FIG. $1 \mathrm{~b}$ centre, arrows). One practical way to test this hypothesis is to scan patients directly after diagnosis $^{6}$ using 7 T UHF MRI M1 quantitative myelin imaging together with functional imaging, in which individuals are asked to move their foot, hand or face ${ }^{2}$. This allows
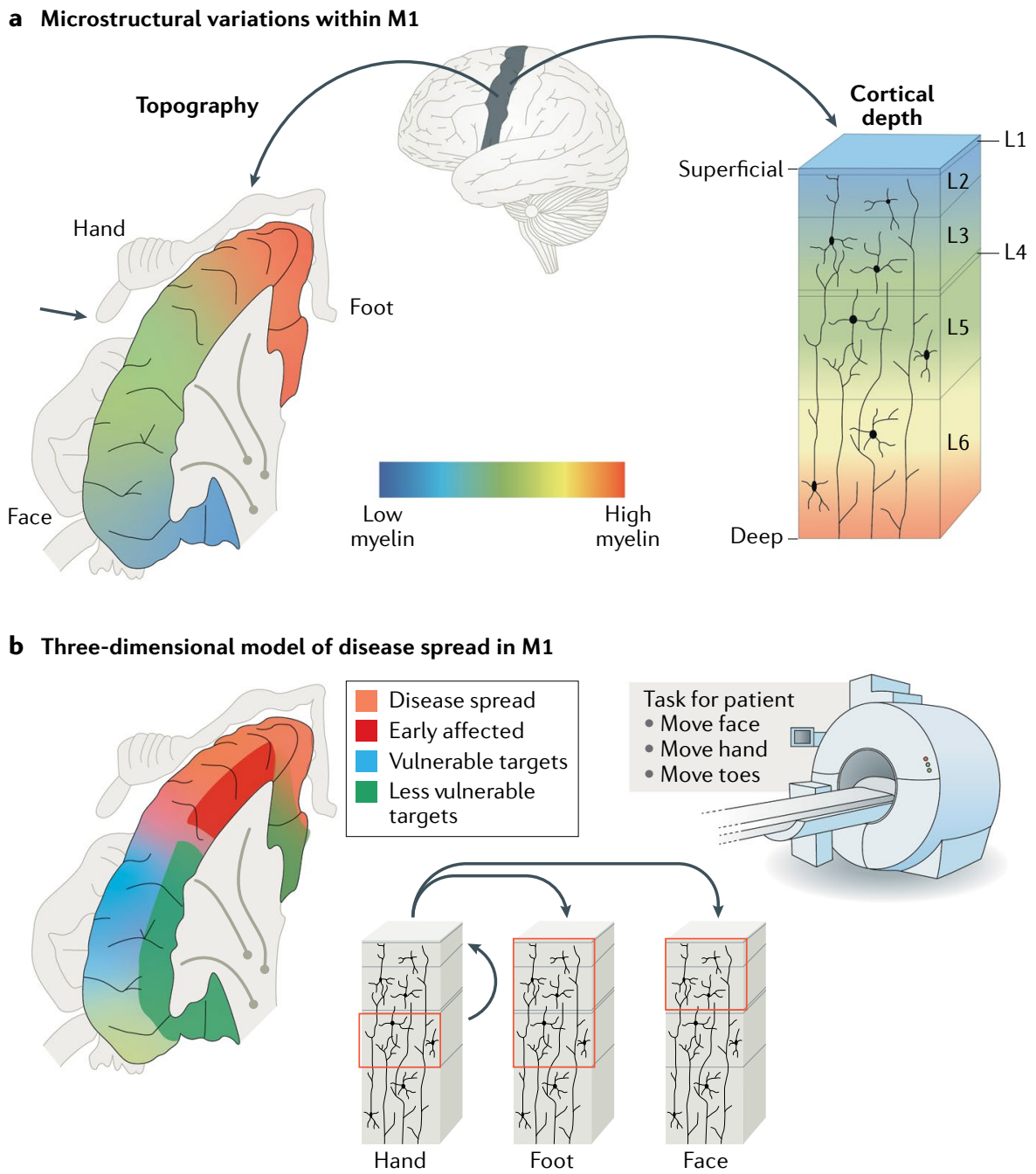

Fig. 1 | Topographical layer imaging in M1. a | The myelination within M1 presents with high heterogeneity even in healthy participants. Two major gradients are microstructural variations in different cortical depth (right), where deeper layers are more myelinated than superficial layers, and microstructural variations along the cortical surface (left), where inferior areas show lower cortical myelin than superior areas. In addition, there are areas of reduced cortical myelin that separate major body part representations such as the hand and the face (marked with an arrow). $\mathbf{b} \mid$ A three-dimensional model of disease spread takes into account both gradients, which allows monitoring of disease spread through cortical depth and across topographical areas. Vertical arrow indicating initial spread from L5 to L2/3, followed by the affection of other cortical areas (horizontal arrows). 
for the identification of layer-specific myelin changes in early-affected, late-affected and non-affected body part areas in M1 and to monitor disease spread body-part-wise and layer-wise in subsequent scans. This approach may offer the possibility to detect M1 microstructural changes at a stage before the disease spreads through L2/3 connections to other topographical areas, or to the contralateral hemisphere, but after the disease has spread from L5 to L2/3, and would thus allow testing the model as proposed by McColgan et al.

In summary, when investigating neurodegenerative disease spread in M1, it is of critical importance to consider local microstructural gradients and the inherent differentiation between topographical areas within M1. Their combined investigation at different clinical stages may allow novel pathophysiological insights into disease spread as well as insights into which individual profiles of the local myeloarchitecture and cell architecture relate to slow versus fast disease progression (that is, factors that constitute microstructural resilience).

There is a reply to this letter by McColgan, P., Joubert, J., Tabrizi, S. J. \& Rees, G. Nat. Rev. Neurosci. https://doi.org/10.1038/s41583-02000405-9 (2020).

Stefanie Schreiber (iD ${ }^{\otimes}$, Alicia Northall (iD) ${ }^{2}$, Miriam Weber ${ }^{1}$, Stefan Vielhaber ${ }^{1}$ and Esther Kuehn 'Department of Neurology, Otto-von-Guericke University Magdeburg, Magdeburg, Germany. ${ }^{2}$ Institute for Cognitive Neurology and Dementia Research, Otto-von-Guericke University Magdeburg, Magdeburg, Germany.

凶e-mail: stefanie.schreiber@med.ovgu.de https://doi.org/10.1038/s41583-020-00404-w

1. McColgan, P., Joubert, J., Tabrizi, S. J. \& Rees, G. The human motor cortex microcircuit: insights for neurodegenerative disease. Nat. Rev. Neurosci. 21, 401-415 (2020)

2. Kuehn, E. et al. Body topography parcellates human sensory and motor cortex. Cereb. Cortex 27 3790-3805 (2017).

3. Glasser, M. F. et al. A multi-modal parcellation of human cerebral cortex. Nature 536, 171-178 (2016).

Kuehn, E. \& Sereno, I. M. Modelling the human cortex in three dimensions. Trends Cogn. Sci. 22, 1073-1075 (2018).

5. Ravits, J. M. \& La Spada, A. R. ALS motor phenotype heterogeneity, focality, and spread: deconstructing motor neuron degeneration. Neurology 73, 805-811 (2009).

6. Hensiek, N. et al. Sonographic and 3T-MRI-based evaluation of the tongue in ALS. Neuroimage Clin. 26, 102233 (2020).

\section{Acknowledgements}

E.K. was funded by ESIF/EFRE 2014-2020; and FKZ: ZS/2016/04/78113, Vorhaben: Center for Behavioral Brain Sciences (CBBS). S.S. and E.K. received funding within the CBBS Neuronetwork initiative (Project Diagnostic Glove). A.N. was funded by the Else Kröner Fresenius Stiftung (EKFS) (2019-A03). This work was further supported by a grant to S.S. and S.V. from the Foundation of Medical Research Frankfurt/Main, Germany. E.K., S.S. and S.V. are affiliated to the German Center for Neurodegenerative Diseases (DZNE) Magdeburg and to the CBBS.

\title{
Reply to 'Topographical layer imaging as a tool to track neurodegenerative disease spread in M1'
}

\author{
Peter McColgan (1), Julie Joubert, Sarah J. Tabrizi(i) and Geraint Rees (1)
}

We would like to thank Stefanie Schreiber, Alicia Northall, Miriam Weber, Stefan Vielhaber and Esther Kuehn for their interest in our Review (The human motor cortex microcircuit: insights for neurodegenerative disease. Nat. Rev. Neurosci. 21, 401-415 (2020)) ${ }^{1}$ and their correspondence (Topographical layer imaging as a tool to track neurodegenerative disease spread in M1. Nat. Rev. Neurosci. https://doi.org/10.1038/s41583020-00404-w (2020)) $)^{2}$. Their comment adds an important technical point about the directionality of myelin gradients. They highlight a seminal study that demonstrates the somatosensory cortex $3 \mathrm{~b}$ and motor cortex 4 are not homogeneous, as suggested by Brodmann ${ }^{3}$, but can be parcellated into sub-fields with clear boundaries, representing hand and face areas. This is eloquently demonstrated using a functional MRI task to identify hand and face areas and 7T MRI to identify myelin borders between these regions at the individual level ${ }^{4}$.

This provides a compelling framework, in which the spread of neurodegenerative disease could not only be tracked across cortical layers but also across body topography, enabling a 3D representation ${ }^{5}$. The authors identify amyotrophic lateral sclerosis (ALS) as disease, which can spread body-part-wise initially across the ipsilateral and then the contralateral hemisphere. Indeed, the latter is in keeping with our model showing loss in neurons in the contralateral hemisphere via the death and axonal dying back of ipsilateral intratelencephalic neurons.

The 3D framework proposed may also be applied to other neurodegenerative diseases that demonstrate selective vulnerability of somatosensory and motor cortices. Idiopathic Parkinson disease, for example, typically has a unilateral onset, initially affecting upper limb and may then progress with lower limb symptoms such as dystonia and eventually affect the contralateral side ${ }^{6}$. Huntington disease can also present with subtle chorea, such as finger flicking in the upper limbs on walking, with more generalized chorea and focal limb dystonia as the disease progresses ${ }^{7}$.
While the framework proposed by Schreiber et al. may provide a unique opportunity to generate layer-specific and topographyspecific measures of motor cortex structure and function, it is not without challenges. As Schreiber et al. highlight, M1 exhibits a surprisingly high heterogeneity even in healthy individuals. This may pose challenges in quantitatively comparing myelin patterns in patients with neurodegenerative disease, where large participant numbers may be required in order to differentiate between naturally occurring population variations in myelin distribution and disease-related effects.

Peter McColgan $1{ }^{1 \otimes}$, Julie Joubert ${ }^{l}$, Sarah J. Tabrizi (iD ${ }^{1,2}$ and Geraint Rees (iD) 3,4 'Huntington's Disease Research Centre, UCL Institute of Neurology, University College London, London, UK.

Dementia Research Centre at UCL, University College London, London, UK.

${ }^{3}$ Wellcome Centre for Human Neuroimaging, UCL Institute of Neurology, University College London, London, UK. ${ }^{4}$ UCL Institute of Cognitive Neuroscience, University College London, London, UK. 凶e-mail:p.mccolgan@ucl.ac.uk https://doi.org/10.1038/s41583-020-00405-9

1. McColgan, P., Joubert, J., Tabrizi, S. J. \& Rees, G. The human motor cortex microcircuit: insights for neurodegenerative disease. Nat. Rev. Neurosci. https://doi.org/10.1038/s41583-020-0315-1 (2020).

2. Schreiber, S. et al. Topographical layer imaging as a tool to track neurodegenerative disease spread in M1. Nat. Rev. Neurosci. https://do org/10.1038/s41583-020-00404-w (2020).

3. Brodmann, K. Vergleichende lokalisationslehre der grosshirnrinde in ihren prinzipien dargestellt auf grund des zellenbaues [German] (Johann Ambrosius Barth, 1909).

4. Kuehn, E. et al. Body topography parcellates human sensory and motor cortex. Cereb. Cortex 27, 3790-3805 (2017).

5. Kuehn, E. \& Sereno, M. I. Modelling the human cortex in three dimensions. Trends Cogn. Sci. 22, 1073-1075 (2018).

6. Kalia, L. V. \& Lang, A. E. Parkinson's disease. Lancet 386, 896-912 (2015).

7. McColgan, P. \& Tabrizi, S. J. Huntington's disease: a clinical review. Eur. J. Neurol. 25, 24-34 (2018).

\section{Competing interests}

The authors declare no competing interests. 